\title{
Molecular cloning and expression of key gene encoding hypothetical DNA polymerase from $B$. mori parvo-like virus
}

\author{
Junhong Zhang*, Guohui Li*, Huiqing Chen, Xiaogang Li, Meng Lv, Keping Chen and Qin Yao \\ Institute of Life Sciences, Jiangsu University, Zhenjiang, China.
}

\begin{abstract}
BmPLV-Z is the abbreviation for Bombyx mori parvo-like virus (China isolate). This is a novel virus with two single-stranded linear DNA molecules, viz., VD1 (6543 bp) and VD2 (6022 bp), which are encapsidated respectively into separate virions. Analysis of the deduced amino acid sequence of VD1-ORF4 indicated the existence of a putative DNA-polymerase with exonuclease activity, possibly involved in the replication of BmPLV-Z. In the present study, a recombinant baculovirus was constructed to express the full length of the protein encoded by the VD1-ORF4 gene (3318 bp). In addition, a 2163-bp fragment amplified from the very same gene was cloned into prokaryotic expression vector pET-30a and expressed in E.coli Rosetta 2 (DE3) pLysS. The expressed fusion protein was employed to immunize New Zealand white rabbits for the production of an antiserum, afterwards used for examining the expression of the protein encoded by VD1-ORF4 gene in Sf-9 cells infected with recombinant baculovirus. Western blot analysis of extracts from thus cells infected revealed a specific band of about $120 \mathrm{kDa}$, thereby indicating that the full length protein encoded by the VD1-ORF4 gene had been successfully and stably expressed in Sf-9 cells.
\end{abstract}

Key words: Bombyx mori parvo-like virus, DNA polymerase, recombinant protein, Sf-9.

Received: April 19, 2010; Accepted: May 31, 2010.

\section{Introduction}

Parvoviruses are small icosahedral, non-enveloped particles $18-26 \mathrm{~nm}$ in diameter, which possess a small linear single-stranded DNA in the genome characterized by inverted terminal repeats (ITRs) and palindromic sequences folding to form a hairpin structure. It is generally thought that DNA synthesis of parvoviruses is initiated by a self-priming mechanism (Tattersall et al., 1973; Astell et al., 1985; Bando et al., 1990). Unlike parvoviruses, the genome of BmPLV-Z is composed of two separated linear single-stranded DNA molecules (VD1 and VD2), each of which is encapsidated separately in virions. The genome was sequenced and published in 2005 (Accession Number DQ017268 and DQ017269, in order to characterize its structure. Sequence analysis showed VD1 consist of 6,543 $\mathrm{nt}$ including inverted terminal repeats (ITRs) of $224 \mathrm{nt}$, and VD2 of 6,022 nt with like repeats of $524 \mathrm{nt}$. A $53 \mathrm{bp}$ consensus sequence was found at both the VD1 and VD2 terminal. Unlike other parvoviruses and densovirus, the ITRs of BmPLV-Z, besides being imperfect, were incapable of forming palindrome structures. Furthermore, whereas VD1 genome contains four ORFs, of which ORF4 located in the genomic right-hand portion encodes a hypothetical DNA

Send correspondence to Yao Qin. Institute of Life Sciences, Jiangsu University, 301\# Xuefu Road, Zhenjiang 212013, China. E-mail: yaoqin@ujs.edu.cn.

*These authors contributed equally to this study. polymerase, and VD2 genome contains two major ORFs (Wang et al., 2007; Li et al., 2009). The unique properties of BmPLV-Z imply a possible different replication mechanism to that of densoviruses or vertebrate parvovirus ( $\mathrm{Li}$ et al., 2009; Tijssen et al., 1995). In fact, based on the analysis of deduced amino acid sequences (Tijssen et al., 1995; Kapitonov et al., 2006), it was concluded that there is a DNA polymerase-like protein encoded by VD1-ORF4 in $B m P L V-Z$, which is not found in densoviruses or vertebrate parvovirus.

Bioinformatic analysis indicated that the N-terminal 600-amino-acid fragment of the VD1-ORF4 protein contains three motifs, Exo IDXEExo II (Nx3F/YD) and Exo III (Yx3D), which are conserved in the 3'-5' exonuclease activity domain of prokaryotic and eukaryotic DNA polymerases (Morrison et al., 1991). Moreover, the fragment 706-1004 of VD1-ORF4 protein contains five motifs, Dx2SLYP (motif I), Kx3NSxYG (motif II), Tx2GxAR (motif III), YxDTDS (motif IV) and KxY (motif V), which are conserved in protein-primed DNA polymerase belonging to the family of B DNA polmerase. Protein-primed DNA polymerase initiate DNA replication by using a protein as a primer (Kapitonov et al., 2006). These polymerases display both 3'-5' exonucleolytic and 5'-3' synthetic activities defined by two structurally independent $\mathrm{N}$ - and $\mathrm{C}$-terminal domains. The five conserved motifs are involved in synthetic activities of polymerase, such as dNTP binding, DNA synthesis and phosphorylation hydrolysis 
(Bernad et al., 1990). Taken together, we propose that the protein encoded by VD1-ORF4 is very likely to be a novel DNA polymerase.

However, there is no experimental evidence showing whether the full length VD1-ORF4 protein is really expressed in insect cells infected with $B m$ PLV-Z. In this study, we constructed a recombinant baculovirus to express the full length protein encoded by the VD1-ORF4 gene (3318 bp) in Sf-9 cells for characterizing the stability of the full length VD1-ORF4 protein. A 2163-bp fragment of BmPLV-Z VD1-ORF4 was cloned into pET-30a and expressed in Rosetta 2 (DE3) pLysS. The amino acid sequence encoded by this fragment contains two exonuclease and five polymerase motifs of the hypothetical DNA polymerase. The $6 \mathrm{xHis}$-tagged fusion protein was confirmed by Western blot and mass spectrographic analysis. The fusion protein expressed in $E$. coli was purified by a $\mathrm{Ni}^{2+}-\mathrm{NTA}$ column (Novagen) and then used to raise an antiserum in rabbits according to the method of Sambrook et al (1989). This antiserum was specially employed to detect the VD1ORF4 protein expressed in Sf-9 cells.

\section{Materials and Methods}

\section{Cells and virus}

Sf-9 cell, TG1 and Escherichia coli Rosetta 2 (DE3) pLysS strains, besides pFasBacHTb and DH10Bac containing the AcMNPV bacmid, were maintained in our laboratory. The AcMNPV $\mathrm{BV}$ stocks were prepared by transfecting Sf-9 cells with extracted recombinant bacmids. TC-100 insect medium was supplemented with $10 \%(\mathrm{v} / \mathrm{v})$ fetal bovine serum (Gibco, USA). Sf-900 II SFM and EXPRES-FIVE SFM were purchased from Invitrogen Company (USA). Restriction enzyme, T4 DNA ligase, and PCR reagents were purchased from TaKaRa Company (Dalian, China). Primers and other reagents were obtained from Shanghai Sangon Bio-technology Corporation (Shanghai, China).

\section{Cloning of BmPLV-Z VD1-ORF4}

The primer pair P1: 5'-CCGGAATTCATGTTTTT AACTGATTTATATAG-3' and P2: 5'-CCGCTCGAGTT ATTCAATTACAACATCATC-3' was designed to amplify the 2163-bp fragment at the 3'-terminal of BmPLV-Z VD1-ORF4. The PCR product was cloned into pMD18-T which generated recombinant plasmid pMD18-T-VD1ORF4 (2163 bp), and was validated by restriction analysis and DNA sequencing.

The primer pair P3: 5'-CCGGAATTCAAATGCC TTTAGTGAAGATTAC-3' and P2: 5'-CCGCTCGAGTT ATTCAATTACAACATCATC-3' was designed to amplify the 3318-bp full length coding region. The PCR product was cloned into pMD18-T which, in turn, generated plasmid pMD18-T-VD1-ORF4 (3318 bp), and was subse- quently validated by restriction analysis and complete sequencing.

\section{Expression of BmPLV-Z VD1-ORF4 (2163 bp) in E.coli and Western blot analysis}

The 2163-bp fragment was excised from pMD18T-VD1-ORF4 (2163 bp) by EcoRI and XhoI, and subsequently subcloned into the pET-30a expression vector (Novagen, USA) in frame with a $6 \mathrm{xHis}$ tag at the $\mathrm{N}$ terminus. The recombinant plasmid, pET-30a-VD1-ORF4 /2163, was verified by restriction analysis and DNA sequencing. RosettaTM 2 (DE3) pLysS cells harboring pET-30a-VD1ORF4/2163 were grown to an OD600 of 0.6 , and then induced by the addition of isopropyl- $\beta$-D-thiogalactopyranoside (IPTG) at concentrations ranging from 0.2 to $0.8 \mathrm{mmol} / \mathrm{L}$. After incubation for $10 \mathrm{~h}$ at $28{ }^{\circ} \mathrm{C}$, cells were harvested by centrifugation at $7000 \mathrm{~g}$ for $15 \mathrm{~min}$ at $4{ }^{\circ} \mathrm{C}$. The fusion protein present in the cells was separated in $10 \%$ SDS-polyacrylamide gels and stained with Coomassie brilliant blue. Western blot analysis, using anti-6xHis tag antibodies, was applied for confirming the presence of $6 \mathrm{xHis}$ tagged fusion proteins. Briefly, this involved resuspending harvest-cell pellets in a SDS-PAGE loading buffer, and their boiling for $10 \mathrm{~min}$, with subsequent analysis. Anti6xHis tag antibodies were used at a dilution of 1:500. Horseradish peroxidase conjugated goat anti-rat IgG was used as secondary antibody. The immunoreactive proteins were visualized by using DAB staining.

\section{Verifying fusion protein expression by way of mass spectrum analysis}

The proteins were excised and digested with trypsin, and then analyzed by matrix-assisted laser desorption ionization-time-of-flight mass spectrometry MALDI-TOF (Bruker Daltonics, Germany). Peptide mass information on tryptic peptides from fusion proteins was obtained, and subsequently used to search corresponding proteins in the NCBI database via the MASCOT program.

\section{Preparation of an antibody against protein fragment VD1-ORF4}

The expressed fusion protein was purified by means of a $\mathrm{Ni}^{2+}$-NTA column (Novagen) and used to raise a polyclonal antibody in New Zealand White rabbit. The rabbit was subcutaneously immunized at four different sites by injecting $200 \mu \mathrm{g}$ of antigen, with complete Freund's adjuvant. Three weeks later, the rabbit was injected with $200 \mu \mathrm{g}$ protein with Freund's incomplete adjuvant as booster injections. Subsequently, two additional booster doses were administered at 3-week intervals. Two weeks after the final dose, serum was separated from blood collected from the rabbit's ear vein, and then stored in $0.1 \%$ sodium azide at $-20{ }^{\circ} \mathrm{C}$. The anti-VD1-ORF4 antibodies were purified using protein-A-sepharose CL-4B (Sigma, USA), whereupon they underwent SDS-PAGE analysis to check their purity. 
Expression of VD1-ORF4 (3318 bp) in Sf-9 cells and Western blot analysis

A Bac-to-Bac baculovirus expression system was used for expressing BmPLV-Z full length VD1-ORF4. Briefly, VD1-ORF4 fragments were excised from pMD18-T-VD1ORF4 with EcoRI and XhoI, and subcloned into $\mathrm{pFasBacHTb}$ to generate pFB-VD1-ORF4. DH10B cells harboring the AcMNPV bacmid (Ac-bacmid) and helper vector encoding a transposase were transformed with donor plasmid pFB-VD1-ORF4, so as to generate recombinant Ac-bacmid-VD1-ORF4 by transposition. E.coli colonies containing recombinant Ac-bacmid-VD1-ORF4 were screened by blue/white selection according to manufacturer's instructions. Sf-9 cells were transfected with either extracted recombinant Ac-bacmid-VD1-ORF4 or wild Acbacmid, and the resultant supernatants of both transfections harvested.

A monolayer of Sf-9 cells was infected with a mock or recombinant virus supernatant (multiplicity of the infectious virus was 5), in order to obtain expression of 6xHisVD1-ORF4 protein. Cells were harvested at $72 \mathrm{~h}$ postinfection. These were first pelleted and resuspended in phosphate-buffered saline (PBS, pH 7.4), then lysed in a SDS - PAGE loading buffer, and finally analyzed in Western blots. VD1-ORF4 protein specific antibodies were used at a dilution of 1:1000. Horseradish peroxidase conjugated goat anti-rabbit IgG was used as secondary antibody. Visualization of immunoreactive proteins was through $\mathrm{DAB}$ staining.

\section{Results}

\section{Identification of the target fragment amplified from BmPLV-Z VD1-ORF4}

A 2163-bp fragment was amplified from BmPLV-Z VD1-ORF4 by the specific primer pair P1 and P2 (Figure 1a). The PCR products obtained were cloned into pMD18$\mathrm{T}$ (Figure $1 \mathrm{~b}$ ) and then sequenced. In addition, the full length of VD1-ORF4 was amplified by the specific primer pair P3 and P2 (Figure 1c). The PCR products obtained were cloned into pMD18-T (Figure 1d) and sequenced. The size of obtained sequences corresponded with the expected fragments amplified from VD1-ORF4.

\section{Construction of the expression plasmid pET-30a-VD1-ORF4/2163}

The plasmid pMD18-T-VD1-ORF4/2163 was digested with EcoRI and XhoI, whereupon the released 2163-bp fragment was purified and ligated with pET-30a, which itself was also digested with the same two enzymes, to generate pET-30a-VD1-ORF4/2163 (Figure 1e).
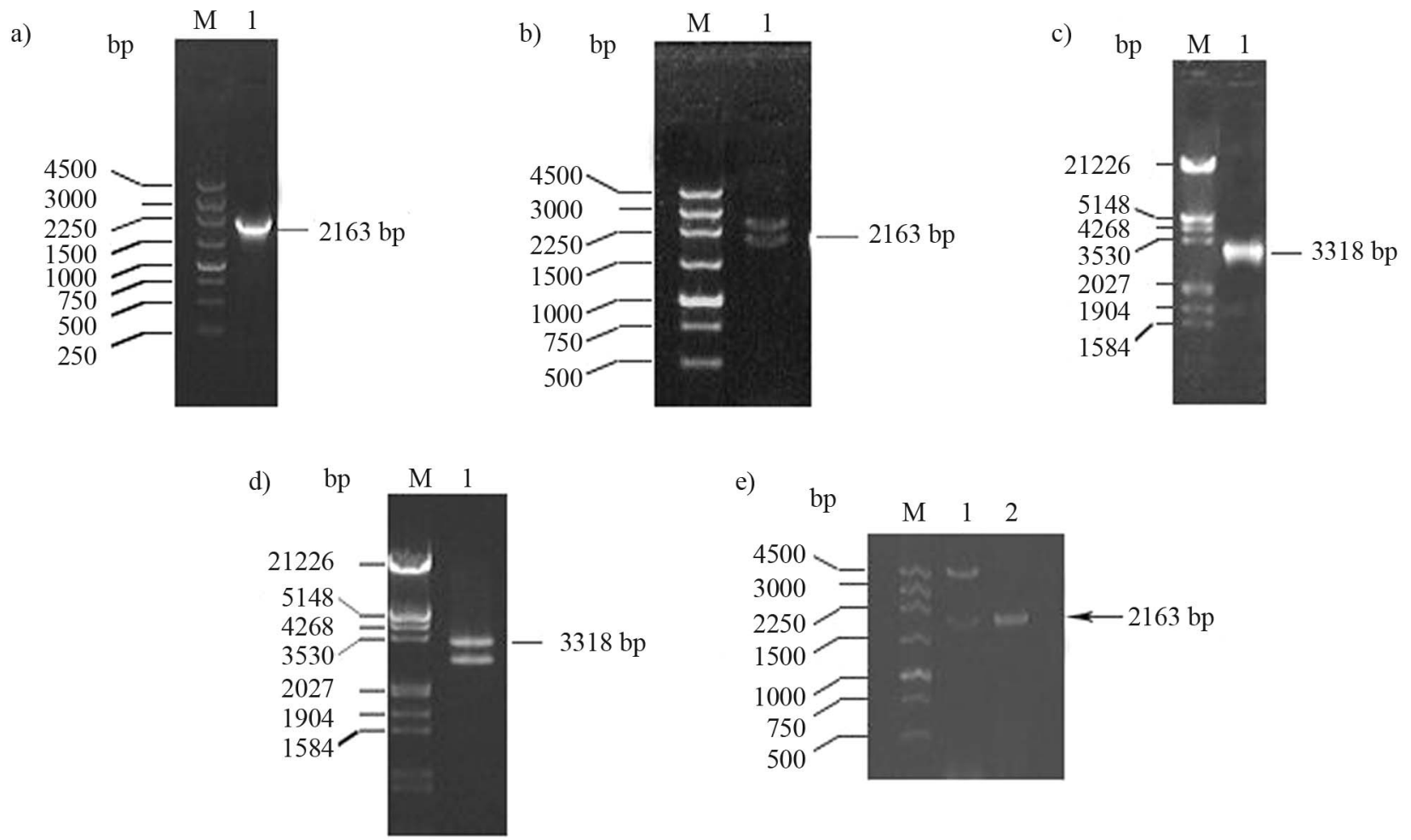

Figure 1 - Agarose gel electrophoresis of PCR products and recombinant plasmids digested with EcoRI and XhoI. (a) Lane M, DNA marker; Lane 1, the 2163-bp fragment product from VD1-ORF4. (b) Lane M, DNA marker; Lane 1, pMD18-T-VD1-ORF4/2163 digested with EcoRI and XhoI. (c) Lane M, DNA marker; Lane 1, the 3318-bp fragment product from VD1-ORF4. (d) Lane M, DNA marker; Lane 1, pMD18-T-VD1-ORF4 digested with EcoRI and XhoI. (e) Lane M, DNA maker; Lane 1, pET-30a-VD1-ORF4/2163 digested with EcoRI and XhoI; Lane 2, the 2163-bp PCR product from VD1-ORF4. 


\section{Expression of fusion protein in E. coli and analysis by Western blot and MS}

SDS-PAGE analysis indicated that target protein expression could be induced by different concentrations of IPTG (Figure 2a). In its absence, expression proved to be basal (Figure 2a Lane 1), whereas it was highest with the addition of $0.8 \mathrm{mmol} / \mathrm{L}$ (Figure $2 \mathrm{a}$ Lane 4). Protein induction was confirmed by Western blot analysis using anti6xHis tag antibody (Figure 2a Lane 5). The fusion-protein band was excised for MS analysis. The Mascot search was carried out with carbamidomethyl as the fixed modification of cysteine and variable N-terminal Gln-pyroGlu. The fusion protein was identified as part of BmPLV-Z VD1-ORF4 based on deduced amino acid sequence (Figure $2 b$ ).

\section{Preparation of Specific antibody against VD1-ORF4}

After confirmation of the 6xHis fusion protein, it was purified and subsequently used to immunize rabbits to raise a specific antibody. The harvested antiserum was further purified by using protein-A-sepharose CL-4B, with posterior SDS-PAGE analysis to check the purity of the final product (Figure 3a).

\section{VD1-ORF4 expression in Sf-9 cells}

The recombinant Ac-bacmid-VD1-ORF4 was confirmed by PCR, using pUC/M13 primers (Figure 3b). A specific band about $5.8 \mathrm{~kb}$ from recombinant Ac-bacmidVD1-ORF4 (Figure 3b Lane 2) was amplified, but only a fragment of $300 \mathrm{bp}$ in the empty Ac-bacmid (Figure 3b a)

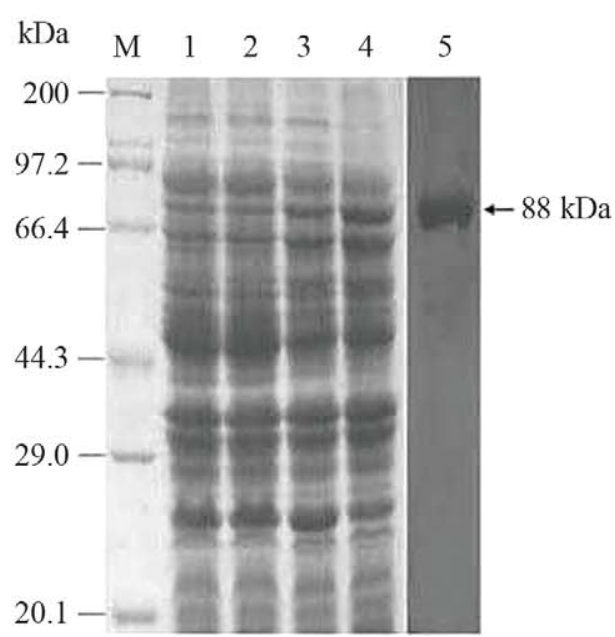

b)

MFLTD

LYSIIKKFQT NDYGKEANLI DEAGERFKCL ACNKNKKGKY YYARHYGLGI FGYYCRSCFL AHNNTFIVYF HNFKGYDHHI LLEQLLNKDS KHNTCRGKSI NKMDVITHKD ILSDFIRITF KDTFNFLPES LASLANKLTT LKYTPDKFKE AFNSGKGEFP YEWFDDFNKL EEIEVPQDPA DWDSRLTNKK GTEEIIKKAN QIWIDNNMQI FHDYVLLYNE LDVWLLLEVF EAFRDTTVNE DKIDPVYFDG APGLTFYLAR MYENSLDMHV IPDKNVYLDV SRNIRGGVTQ VVTKYANIED VDETIVYLDV NTMYSYCMKQ KLANKYLGTL DALPDNYDSD DNFCYFIKGD FSYPEYLHDL PAHLSMPLMP HQYNNKLCTT FLDKKDMLIH SKVFKYYLSK GLVCDKIHYV YKFKQEYIIK DYVETNIQKR NSSTDPGTKD YYKLKNNALF GKTCENVFKY KIFSVTNVNS GDRENKCMSK AKSHITLGNC ILYEECVTRY LLDKPIQIGF TILELAKLMI YEFIYELFDV IPEGSTATML YTDTDSVIFK FKGFNGVHPY KYLLTTSLAS KLDIPINKDG SFGSATKTPG LWSDDTKYKT ITEFIGLRAK QYAYSIANDR DILKHKGIPK NALKDDNNPM NVNDFRNVLF EMKDLTVNIA QIRATKNVLT STVSKKLALS TKDNKRITYT DKVTTLPFGY KGELYSNYMD DVVIE

Figure 2 - Identification of induced target-protein expression in E. coli Rosetta TM 2 (DE3) pLysS, together with Western blot and MALDI-TOF analysis of the induced protein. (a) SDS-PAGE and Western blot analysis of the target protein induced in RosettaTM 2 (DE3) pLysS. Lane M, protein marker; Lane 1, RosettaTM 2 (DE3) plysS with pET-30a-VD1-ORF4/2163 induced without IPTG; Lane 2-4, Rosetta TM 2 (DE3) plysS with pET-30aVD1-ORF $4 / 2163$ induced by IPTG concentrations of $0.2,0.5,0.8 \mathrm{mmol} / \mathrm{L}$, respectively; Lane 5 , the target protein was examined with an antibody against the 6xHis tag. (b) Peptide sequences identified by mass spectrometry. The BmPLV-Z VD1-ORF4 deduced amino acid sequence is shown, and the matched peptide sequences are indicated as red characters.

a)

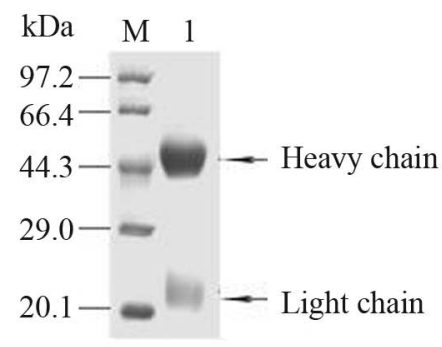

b)

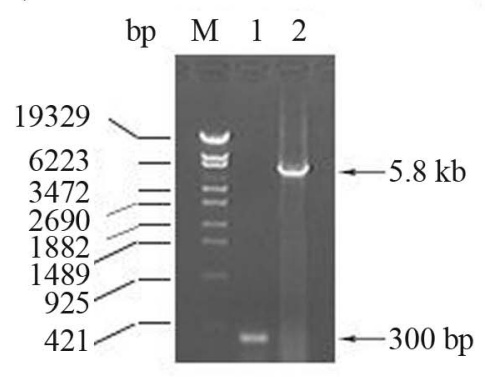

c)

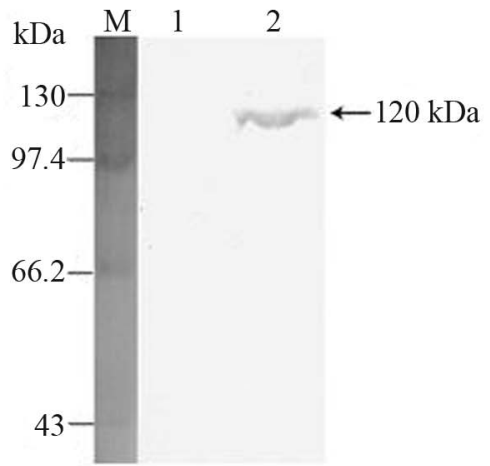

Figure 3 - SDS-PAGE analysis of the purified antibody against VD1-ORF4, electrophoretic analysis of Ac-bacmid-VD1-ORF4 after PCR with pUC/M13 primers, and Western blot analysis to detect VD1-ORF4 expression in Sf-9 cells. (a) Lane M, protein marker; Lane 1, purified polyclonal antibody. The two chains of purified antibody are respectively indicated by an arrow on the right. (b) Lane M, DNA marker; Lane 1, PCR product amplified from wild Ac-bacmid; Lane 2, PCR product amplified from recombinant Ac-bacmid-VD1-ORF4. (c) Lane M, pre-staining protein marker; Lane 1, a protein sample from Sf-9 cells infected with the wild virus was used as control; Lane 2, the target protein was examined with VD1-ORF4 protein specific antibody. 
Lane 1). 6xHis fusion protein expression in Sf-9 cells infected with the recombinant virus was confirmed by Western Blot analysis, using VD1-ORF4 protein-specific antibodies (Figure 3c). Apparently, only a specific band of about $120 \mathrm{kDa}$ was detected in extracts from recombinant virus infected cells (Figure 3c Lane 2), whereas no similar band was detected in extracts from wild virus infected cells (Figure 3c Lane 1), thereby indicating successful expression of the entire VD1-ORF4 of BmPLV-Z and full length stability of the protein in Sf-9 cells.

\section{Discussion}

$B m$ PLV-Z is a viral agent that causes flacherie disease in silkworms, thus adverse to sericulture. The BmPLV-Z genome has been sequenced by Wang et al. (2007). In order to explore the interactions between BmPLV-Z and Bombyx mori, there is the urgent need to study the functions of viral genes, especially the VDIORF4 gene encoding a putative DNA polymerase.

There is a significant difference in genome structure between BmPLV-Z and parvoviruses. It is generally accepted that vertebrate parvovirus genomes contain two major blocks of open-reading frames in the same strand, the left-hand region encoding non-structural proteins and the right structural proteins (Srivastava et al., 1983; Cotmore and Tattersall, 1986, 1987; Alexandersen et al., 1988). However, the BmPLV-Z genome consists of two linear single-strand molecules, VD1 and VD2. The sequence of VD1 is quite different from that of VD2, although they share a common terminal sequence of $53 \mathrm{nt}$ at their 5 '-ends, which facilitates their forming panhandles. Moreover, there is high homology between the deduced amino acid sequence of VD1-ORF4 and various protein-primed DNA polymerases (Kapitonov and Jurka, 2006). It has been stated that replication in Bombyx mori parvo-like viruses (Yamanashi isolate) and adenoviruses is alike (Tijssen and Bergoin, 1995). Accordingly, it is very likely that the protein encoded by VD1-ORF4 is directly involved in virus replication. However, so far, very little is known about the exact replication mechanism of $B m P L V-Z$. To further investigate this mechanism, it is essential to characterize the protein encoded by BmPLV-Z VD1-ORF4.

In this study, a 2163-bp fragment was amplified from BmPLV-Z VD1-ORF4 and expressed in E.coli Rosetta TM 2 (DE3) pLysS. SDS-PAGE analysis revealed the optimal induction conditions to be $0.8 \mathrm{mmol} / \mathrm{L}$ of IPTG for $10 \mathrm{~h}$. Nevertheless, basal expression of the desired fragment also occurred in non-induced cells, thus possibly severely affecting host-cell growth, thereby leading to relatively low expression. Originally, E.coli BL21 (DE3) was used as the host expression strain in attempt to detect the target protein, but without success. Furthermore, analysis of rare codon distribution (data not shown) indicated that BL21 (DE3) was unsuitable for target protein expression, which suggested the importance of the choice of host bacteria. The re- combinant fusion protein was purified on a $\mathrm{Ni}^{2+}$-NTA column and used to raise an antiserum in rabbits. The harvested antiserum was purified and used for further research.

Recombinant baculoviruses are widely used to express heterologous genes in cultured insect cells and insect larvae. The baculovirus expression vector system (BEVS) is particularly advantageous for large scale application $(\mathrm{Hu}$, 2005; Kost et al., 2005). Furthermore, the host of BmPLV$\mathrm{Z}$ is an insect. Thus, studies of BmPLV-Z VD1-ORF4 expression and its function in Sf-9 cells are essential for knowing the physiological role of BmPLV-Z VD1-ORF4 in infection. Full length BmPLV-Z VD1-ORF4 expression in Sf-9 cells was brought about by using a recombinant baculovirus. A specific band of about $120 \mathrm{kDa}$ in length was detected in Sf-9 cells infected with recombinant baculovirus, whereas no smaller-sized bands were observed through Western blot analysis, thus indicating that the full length of VD1-ORF4 protein in these cells was stable. However, as putative DNA polymerase might only be involved in BmPLV-Z replication, further studies of its biochemical function in the infection process are called for.

When starting, production of the target protein was relatively meager, through being only visible by the detection of a specific antiserum (data not shown). Many trial tests were undertaken in order to discover the optimal conditions for reaching a higher level of target protein expression. Thus, a certain number of commercially-available culture media, such as Sf-900 II SFM and EXPRES-FIVE SFM, were adapted for Sf-9 cell growth. Surprisingly, the expression level of the target protein was increased after replacing TC-100 by Sf-900 II SFM, thus laying a solid foundation for its purification for functional and structural studies.

The results obtained in this work, together with previous findings, will be very useful in subsequent studies, such as characterization of the VD1-ORF4 protein and the in vivo identification of its interacting proteins, thereby constituting a step forward in the precise elucidation of the molecular mechanism of VD1-ORF4 gene action in the life cycle of BmPLV-Z.

\section{Acknowledgments}

This work was supported by the National Natural Science Foundation of China (30871826) and the Senior Talent Start Fund (09JDG057).

\section{References}

Alexandersen S, Bloom ME and Perryman S (1988) Detailed transcription map of Aleutian mink disease parvovirus. J Virol 62:3684-3694.

Astell CR, Chow MB and Ward DC (1985) Sequence analysis of the termini of virion and replicative forms of minute virus of mice DNA suggests a modified rolling hairpin model for autonomous parvovirus DNA replication. J Virol 54:171-177. 
Bando H, Choi H, Ito Y and Kawase S (1990) Terminal structure of a densovirus implies a hairpin termini transfer replication which is similar to the model for AAV. Virology 179:57-63.

Bernad A, Lázaro JM, Salas M and Blanco L (1990) The highly conserved amino acid sequence motif Tyr-Gly-Asp-ThrAsp-Ser in alpha-like DNA polymerases is required by phage phi 29 DNA polymerase for protein-primed initiation and polymerization. Proc Natl Acad Sci USA 87:4610-4614.

Cotmore SF and Tattersall P (1986) Organization of nonstructural genes of the autonomous parvovirus minute virus of mice. $\mathrm{J}$ Virol 58:724-732.

Cotmore SF and Tattersall P (1987) The autonomously replicating parvoviruses of vertebrates. Adv Virus Res 33:91-174.

$\mathrm{Hu}$ YC (2005) Baculovirus as a highly efficient expression vector in insect and mammalian cells. Acta Pharmacol Sin 26:405416.

Kapitonov VV and Jurka J (2006) Self-synthesizing DNA transposons in eukaryotes. Proc Natl Acad Sci USA 103:45404545 .

Kost TA, Condreay JP and Jarvis DL (2005) Baculovirus as versatile vectors for protein expression in insect and mammalian cells. Nat Biotechnol 23:567-575.

Li G, Sun C, Zhang J, He Y, Chen H, Kong J, Huang G, Chen K and Qin Y (2009) Characterization of Bombyx mori parvo- like virus non-structural protein NS1. Virus Genes 39:396402.

Morrison A, Bell JB, Kunkel TA and Sugino A (1991) Eukaryotic DNA polymerase amino acid sequence required for 3' $\rightarrow 5$ ' exonuclease activity. Proc Natl Acad Sci USA 88:94739477.

Sambrook J, Fritsch EF and Maniatis T (1989) Molecular Cloning: A Laboratory Manual. 2nd edition. Cold Spring Harbor Laboratory Press, New York, 1659 pp.

Srivastava A, Lusby EW and Berns KI (1983) Nucleotide sequence and organization of the adeno-associated virus 2 genome. J Virol 45:555-564.

Tattersall P, Crawford LV and Shatkin AJ (1973) Replication of the parvovirus MVM II. isolation and characterization of intermediates in the replication of the viral deoxyribonucleic acid. J Virol 12:1446-1456.

Tijssen P and Bergoin M (1995) Densonucleosis viruses constitute an increasingly diversified subfamily among the parvoviruses. Semin Virol 6:347-355.

Wang YJ, Yao Q, Chen KP, Wang Y, Lu J and Han X (2007) Characterization of the genome structure of Bombyx mori densovirus (China isolate). Virus Genes 35:103-108.

Associate Editor: Carlos F.M. Menck

License information: This is an open-access article distributed under the terms of the Creative Commons Attribution License, which permits unrestricted use, distribution, and reproduction in any medium, provided the original work is properly cited. 